Ann. Biol. anim. Bioch. Biophys., 1971, 11 (4) 559-567.

\title{
DIFFÉRENCE GÉNETTIQUE \\ DANS LA DÉCHARGE OVULANTE (LH) \\ CHEZ DES BREBIS DE RACE ILE-DE-FRANCE; RELATIONS AVEC LE NOMBRE D'OVULATIONS
}

\section{J. THIMONIER et J. PELLETIER}

avec la collaboration technique de C. Cornu, Christiane Foucher, Brigitte et G. FounleuL

\author{
Station de Physiologie de la Reproduction, \\ Centre de Recherches de Tours, I. N. R. A., \\ 37 - Nouzilly
}

\section{RÉSUMÉ}

Les caractéristiques de la décharge de LH avant l'ovulation ont été étudiées par dosage radioimmunologique de la LH plasmatique chez 35 brebis de race Ile-de-France provenant de deux troupeaux.

La quantité de LH déchargée, la durée du pic de décharge, les taux plasmatiques maximaux observés ne diffèrent pas en fonction du nombre d'ovulations.

En revanche, ces paramètres diffèrent hautement significativement $(P<0,0 I)$ suivant que les brebis proviennent de l'un ou l'autre troupeau. En particulier, les taux plasmatiques maximaux sont deux fois plus élevés chez les femelles du troupeau I que chez celles du troupeau II (tabl. 5 et fig. $r$ ).

Ces résultats indiquent qu'il existe des différences génétiques selon les souches au sein d'une même race dans le fonctionnement hypothalamo-hypophysaire.

En outre, nous mettons en évidence une différence en fonction du nombre d'ovulations de la durée de l'intervalle entre le début de l'œstrus et celui de la décharge : cet intervalle est. significativement plus élevé chez les femelles ayant deux ovulations $(\mathrm{P}<0,02)$.

Il en ressort donc que le taux d'ovulation ne dépend pas de l'importance et de la durée de la décharge de $\mathrm{LH}$.

\section{INTRODUCTION}

Les études de la décharge préovulatoire de $\mathrm{LH}$ chez la brebis ont toutes été effectuées sur de petits nombres de femelles et 1'importance de cette décharge n'a généralement pas été reliée à un phénomène physiologique autre que le comporte- 
ment d'œstrus (PELIETIER et al., Ig68 $a$; GESChWIND et DEWEY, I968; NISWENDER et al., I968; GoDING et al., I969; WHEATLEY et RADFORD, I969).

Cependant, nous avons indiqué qu'il semblait exister une relation entre la quantité de $\mathrm{LH}$ déchargée et le nombre d'ovulations : aux plus petits pics de LH plasmatique correspondaient les cas d'une seule ovulation (PELIETIER et THIMONIER, I969).

Nous avons voulu vérifier cette hypothèse sur un grand nombre d'animaux et examiner de plus s'il n'existe pas des différences dues à d'autres facteurs, tels que l'origine génétique des femelles. A cet effet, une expérience a été conduite chez des brebis d'une même race, mais appartenant à deux troupeaux ayant des taux d'ovulations comparables.

\section{MATÉRIEL ET MÉTHODES}

49 brebis adultes de race Ile de France provenant de deux troupeaux distincts (I et II) ont été utilisées pendant la saison sexuelle (septembre). Les poids des animaux des deux troupeaux étaient voisins.

Dans cette race, le taux d'ovulations multiples est voisin de $50 \mathrm{p}$. Ioo. On pouvait donc espérer une répartition égale entre les femelles ayant une seule ovulation et celles ayant au moins deux ovulations.

Afin d'effectuer une observation précise des ostrus pendant une courte période et de limiter celle des prélèvements sanguins, l'cestrus et l'ovulation ont été synchronisés à l'aide d'un traitement progestatif chez la totalité des femelles (RoBINsoN, r965). Les prélèvements sanguins ont été réalisés, non pendant l'œestrus induit par ce traitement, mais lors de l'oestrus suivant. On peut considérer que l'effet du progestagène est alors négligeable (FAULKNER et HopwOOD, I967) bien que la synchronisation des cycles œestriens soit conservée.

\section{I. - Détection des chaleurs}

Le contrôle du comportement d'œestrus à l'aide de béliers entiers munis de tabliers (MaULton et DAUZIER, 1965) a débuté 17,5 jours après l'arrêt du traitement progestatif. Les femelles en cestrus à ce moment ont été éliminées.

La détection de l'œstrus a été faite alors toutes les deux heures en laissant le bélier présent parmi les brebis pendant une heure jusqu'à ce que 35 femelles aient été détectées en cestrus.

Au fur et à mesure de leur venue en cestrus, les brebis ont été transférées dans un local où elles n'ont subi alors que les prélèvements sanguins.

\section{2. - Prélèvements de sang}

Sur toutes les femelles, le premier prélèvement sanguin dans la jugulaire (5 ml sur $25^{\circ}$ UI d'héparine) a été fait exactement 17,5 jours après l'arrêt du traitement progestatif. Ils se sont poursuivis ensuite toutes les 4 heures sur les femelles non encore détectées en cestrus puis toutes les 2 heures pendant 36 heures à partir du début observé de l'oestrus. $-\mathrm{I} 5^{\circ} \mathrm{C}$.

Les plasmas ont été immédiatement prélevés après centrifugation à $4^{\circ} \mathrm{C}$ puis conservés à

\section{3. - Expression des résultats}

Les dosages de LH plasmatique ont été réalisés par radioimmunologie (PELLETIER et al., rg68 b). Les résultats sont exprimés en nanogrammes de $\mathrm{LH} \mathrm{M}_{1} / \mathrm{ml}$ de plasma de sang périphérique( $\left.{ }^{1}\right)$.

L'importance de la décharge ovulante a été estimée par les concentrations maximales observées $(\mathrm{ng} / \mathrm{ml})$ et par des mesures planimétriques des représentations graphiques des pics de $\mathbf{L}$.

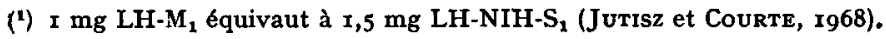


Arbitrairement, nous avons considéré que la décharge ovulante débutait lorsque le taux de LH est supérieur à $6 \mathrm{ng} / \mathrm{ml}$ de plasma. Cette référence a servi aux calculs de la durée et de la surface des pics de LH.

$$
\text { 4. - Mesure du taux d'ovulation }
$$

Au terme de l'expérience, le nombre d'ovulations a été contrôlé par cœlioscopie chez tous les animaux.

\section{RÉSULTATS}

Pendant la durée des observations, 2 I brebis du troupeau I et I4 brebis du troupeau II sont venues en œstrus. Les résultats présentés se réfèrent à ces 35 animaux.

\section{I. - Taux d'ovulations}

Toutes les brebis ont ovulé : I7 ont eu une seule ovulation et $\mathrm{I} 8$, deuxovulations. Cette répartition des animaux permet donc d'analyser les caractéristiques de la décharge de $\mathrm{L} / \mathrm{H}$ en fonction du nombre d'ovulations.

Le tableau I indique les pourcentages respectifs des femelles de chacun des troupeaux ayant eu un ou deux corps jaunes : les brebis du troupeau I ont un taux d'ovulation légèrement supérieur à celles du troupeau II, mais cette différence n'est pas significative.

TABLEAU I

Pourcentages des femelles des troupeaux $I$ et II ayant une ou deux ovulations

\begin{tabular}{l|c|c|c|c}
\hline \hline \multirow{2}{*}{$\begin{array}{c}\text { Nombre } \\
\text { d'ovulations }\end{array}$} & \multicolumn{2}{|c}{1} & \multicolumn{2}{|c}{2} \\
\cline { 2 - 4 } & Nombre de brebis & P. 100 & Nombre de brebis & P. 100 \\
\hline Troupeau I & 9 & 42,9 & 12 & 57,1 \\
\hline Troupeau II & 8 & 57,1 & 6 & 42,9 \\
\hline \hline
\end{tabular}

\section{2. - Caractéristiques de la décharge de $L H$}

a) Importance de la décharge ovulante.

Les surfaces moyennes des pics représentant la décharge ovulante chez les brebis des 2 troupeaux dans les cas de une ou deux ovulations sont présentées dans le tableau 2

Il ressort une différence importante entre les animaux provenant du troupeau I et ceux du troupeau II sans que le nombre d'ovulations ait un effet apparent. Ainsi, la surface moyenne du pic de $\mathrm{L}_{6} \mathrm{H}$ chez les brebis du troupeau II représente environ 55 p. roo de celle des brebis du troupeau I.

Après transformation logarithmique des données, 1'analyse de variance indique que cette différence est très hautement significative (tab1. 3 ). 
En tenant compte de ce résultat l'analyse de variance ne permet pas de mettre en évidence une différence significative quant à l'importance de la décharge ovulante suivant que les brebis ont eu une ou deux ovulations. ( $F=0,5$ pour 34 degrés de liberté).

\section{TABLEAU 2}

Importance de la décharge de $L H$

$\left(\mathrm{mm}^{2} \pm \mathrm{S}_{m}\right)$

\begin{tabular}{c|c|c}
\hline \hline Nombre d'ovulations & 1 & 2 \\
\hline Troupeau I & $3503 \pm 105$ & $3846 \pm 261$ \\
\hline Troupeau II & $2083 \pm 111$ & $1966 \pm 164$ \\
\hline
\end{tabular}

Les calculs ont été effectués sur les mesures des aires (au-dessus de $6 \mathrm{ng} / \mathrm{ml}$ ) représentant graphiquement les pics de décharge de LH.

- en abscisse : $10 \mathrm{~mm}=3$ heures,

- en ordonnée : $10 \mathrm{~mm}=4 \mathrm{ng} / \mathrm{ml}$.

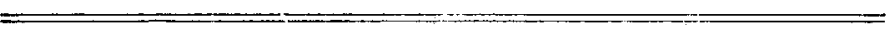

TABLEAU 3

Importance de la décharge de LH. Différence entre troupeaux Analyse de variance après transformation logarithmique

\begin{tabular}{c|c|c|c}
\hline Source de variation & $\begin{array}{c}\text { Somme } \\
\text { des carrés }\end{array}$ & $\begin{array}{c}\text { Degrés } \\
\text { de liberté }\end{array}$ & $\mathrm{F}$ \\
\hline Entre troupeaux & 0,628457 & 1 & $32,21^{* * *}$ \\
\hline Entre brebis & 0,643867 & 33 & - \\
\hline Totaux & 1,272324 & 34 \\
\hline *** $\mathrm{P}<0,001$.
\end{tabular}

b) Début de la décharge de $L H$.

La durée du pic est la période pendant laquelle le taux de $\mathrm{L} / \mathrm{H}$ plasmatique est supérieur à $6 \mathrm{ng} / \mathrm{ml}$. Elle varie de 9 à $\mathrm{I} 7$ heures selon les individus; mais les moyennes des 4 groupes de femelles sont comprises entre Io,9 et I4,2 heures (tabl. 4).

L'analyse de variance montre que la durée de la décharge ne diffère pas selon que les brebis ont eu une ou deux ovulations mais elle révèle une différence hautement significative entre troupeaux (I et II, $\mathrm{P}<0$,or). 
TABLEAU 4

Durée de la décharge de LH

(heures $\pm \mathrm{S}_{m}$ )

\begin{tabular}{c|c|c} 
Nombre d'ovulations & 1 & 2 \\
\hline Troupeau I & $14,2 \pm 1,44$ & $13,6 \pm 0,51$ \\
\hline Troupeau II & $10,9 \pm 0,55$ & $11,8 \pm 1,11$ \\
\hline
\end{tabular}

La durée moyenne de la décharge de $\mathbf{L H}$ chez les femelles du troupeau I est hautement significativement plus grande que chez celles du troupeau II $(\mathrm{p}<0,01)$.

c) Taux maximum de LH plasmatique.

Comme précédemment, il n'y a pas de relation entre les taux maximaux de $L H$ plasmatique et le taux d'ovulation (tabl. 5). En revanche, ils sont environ 2 fois moins élevés chez les femelles du troupeau II que chez celles du troupeau I.

L'analyse de variance montre que cette différence est très hautement significative ( $\mathrm{F}=27,7$ pour 34 degrés de liberté).

TABLEAU 5

Taux maximum de LH plasmatique

$\left(\mathrm{ng} / \mathrm{ml} \pm \mathrm{S}_{m}\right)$

\begin{tabular}{c|c|c}
\hline Nombre d'ovulations & 1 & 2 \\
\hline Troupeau I & $95,2 \pm 10,51$ & $102,0 \pm 6,77$ \\
\hline Troupeau II & $55,3 \pm 8,64$ & $52,7 \pm 7,65$ \\
\hline
\end{tabular}

La moyenne des taux maximaux chez les femelles du troupeau I est très hautement significativement supérieure à celle des femtelles du troupeau II, $P<0,001$.

3. - Intervalle entre le début du comportement d'cestrus et le début de la décharge de $\mathrm{LH}$

68,6 p. Ioo des brebis ont été détectées pour la première fois en cestrus entre I2 et 24 heures. Ce pourcentage est à rapprocher de celui trouvé (62,I p. Ioo) par Cognie, Mariana et Thimonier, (I970).

Dans 33 cas sur 35, la décharge ovulante a débuté après le début du comportement d'œstrus et dans deux cas seulement avant l'apparition de l'œstrus. 


\section{TABLEAU 6}

Intervalle entre le début du comportement d'cestrus et le début de la décharge de $L H$ (heures $\pm \mathrm{S}_{m}$ )

\begin{tabular}{c|c|c}
\hline $\begin{array}{c}\text { Nombre d'ovulations } \\
\text { Troupeau I }\end{array}$ & 1 & 2 \\
\hline Troupeau II & $-\frac{3,2 \pm 1,75}{7,3 \pm 1,16}$ & $7,8 \pm 1,08$ \\
\hline
\end{tabular}
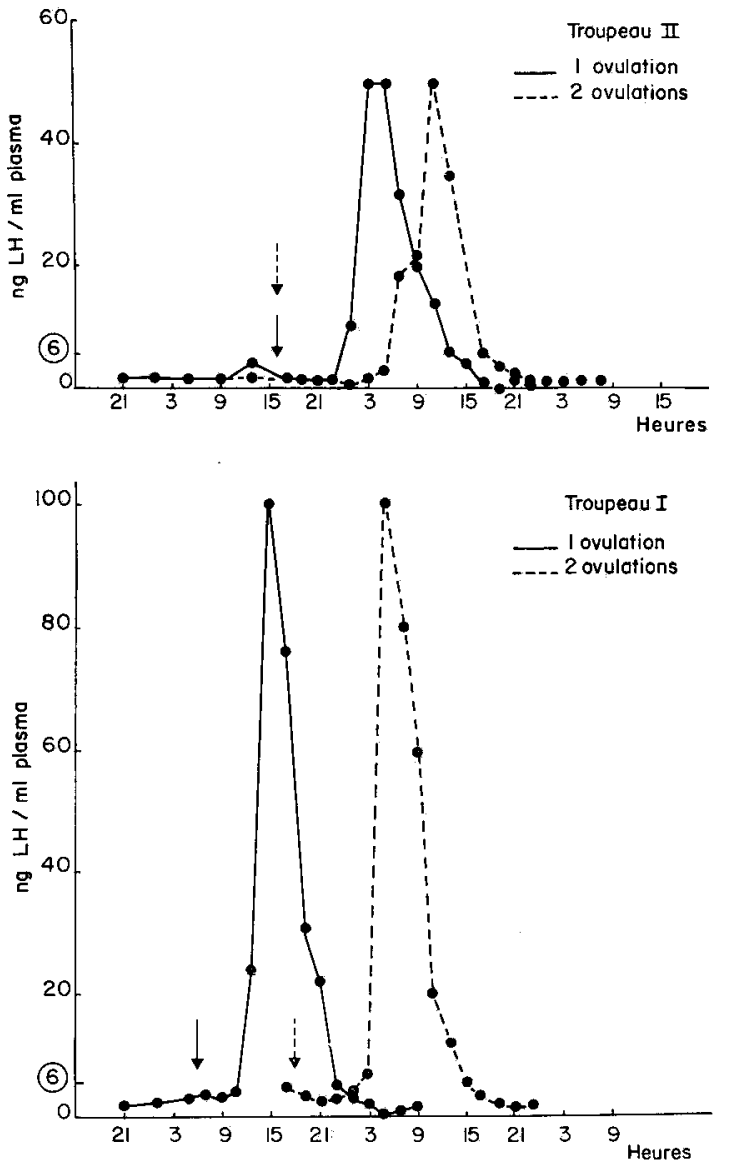

FIG. I. - Décharge de LH dans le sang chez des brebis prêsentant approximativement les caractéristiques moyennes de chacun des 4 lots suivants:

$$
\text { Troupeau I }\left\{\begin{array} { l } 
{ \text { I ovulation } } \\
{ 2 \text { ovulations } }
\end{array} \quad \text { Troupeau II } \left\{\begin{array}{l}
\text { I ovulation } \\
2 \text { ovulations }
\end{array}\right.\right.
$$

On remarque que les intervalles entre le début de l'œstrus (flèche) et celui de la décharge sont plus longs dans les cas de 2 ovulations. 
Les intervalles moyens entre ces deux paramètres pour chacun des 4 groupes de femelles sont présentés dans le tableau 6 .

L'analyse de variance montre que l'intervalle entre le début observé de l'œstrus et celui de la décharge de LH ne diffère pas selon les troupeaux; en revanche, ce paramètre est significativement plus élevé $(P<0,02)$ chez les femelles présentant deux ovulations que chez celles n'en ayant qu'une seule (fig. I).

\section{DISCUSSION}

Les pourcentages de femelles ayant eu une ou deux ovulations (respectivement $48,6$ et $5 I, 4)$ sont favorables à la mise en évidence d'une relation éventuelle entre des caractéristiques de la décharge de $\mathrm{LH}$ et le taux d'ovulation.

Il apparaît clairement que la quantité de LH déchargée n'a pas une influence primordiale sur le taux d'ovulation. En effet, ni les concentrations maximales observées de $\mathrm{L}_{\mathrm{H}}$ plasmatique, ni la durée de la décharge, ni la surface des représentations graphiques des pics de $\mathrm{LH}$ ne diffèrent significativement selon le nombre d'ovulations

En revanche, lorsqu'on compare les valeurs de ces trois caractéristiques chez les femelles des deux troupeaux, on constate qu'elles sont toujours supérieures $(P<0,0 I)$ chez les brebis du troupeau I. En particulier, la surface moyenne des pics de décharge de LH est environ deux fois plus élevée chez les femelles du troupeau I que chez celles du troupeau II.

MaUtíon et Pelietier (I964) ont déjà montré qu'il existait des différences au niveau de la synthèse et de la décharge des hormones gonadotropes entre trois lignées de rats appartenant toutes à la race Wistar. Nous démontrons ici que l'importance de a décharge de $L H$ peut être différente entre les groupes de femelles d'une même race ovine. Les variations importantes des concentrations maximales de $\mathrm{L}_{\mathrm{H}} \mathrm{H}$ dans le plasma pendant l'œstrus ( 46 à $460 \mathrm{ng} / \mathrm{ml}$ ) observées par WHEATLEY et RADFORD (I969) ont peut-être une origine génétique, ces auteurs ayant utilisé deux races d'animaux.

A l'inverse des autres caractéristiques de la décharge de $L_{H} H$, les intervalles moyens entre le début de l'œstrus et celui de la décharge ne diffèrent pas statistiquement entre les femelles des deux troupeaux ; cependant, cet intervalle parait être plus court (o,Io $<\mathbf{P}<0,05)$ chez les femelles du troupeau I. En outre, ces intervalles présentent une analogie remarquable : ils sont significativement plus longs $(P<0,02)$ chez les brebis ayant deux ovulations que chez celles en ayant une seule, quel que soit le troupeau. Ces valeurs que nous avons obtenues sont généralement supérieures à celles indiquées par NisWENDER et al. (I968) et WHEATLEY et RADFORD (I969). Ceci s'explique compte tenu des méthodes de détection de l'œstrus employées.

Dans le cas de notre expérience, nous pouvons accorder aux différences trouvées entre les intervalles une grande fiabilité car un seul critère d'œstrus a été utilisé (immobilité de la brebis lors du chevauchement par le mâle) et la détection a été faite par deux observateurs expérimentés.

Il est vraisemblable que l'intervalle de temps entre le début de l'œstrus et celui de la décharge ait une grande importance sur le taux d'ovulation puisque pour les 
femelles de chacun des troupeaux au nombre d'ovulations le plus élevé correspond l'intervalle le plus grand.

Cela pourrait expliquer que les femelles du troupeau II en dépit d'une décharge significativement moindre ont un taux d'ovulation voisin de celui du troupeau I puisque l'intervalle chez les femelles du troupeau II parait le plus long.

Reçu pour publication en mai 1971

\section{SUMMARY}

\section{GENETIC DIFFERENCE IN THE PREOVULATORY DISCHARGE \\ OF L,H IN TWO FLOCKS OF ILE-DE-FRANCE SHEEP \\ AND ITS REI,ATIONSHIP TO THE NUMBER OF OVA RELEASED}

The characteristics of the preovulatory discharge of $\mathrm{LH}$ have been studied in 35 Ile-de-France ewes derived from two different flocks.

In 48.6 p. Ioo of the ewes, one ovum and in 5 I.4 p. Ioo, two ova were released at ovulation. This distribution would have been suitable for demonstrating any relationship between $L H$ release and the number of ova.

Within each flock the quantity of $\mathrm{LH}$ discharged, the duration of the peak of discharge, and the maximum plasma level did not vary with the number of ova released. However, between the two flocks there were highly significant differences in each variable $(\mathrm{P}<0 . \mathrm{OI})$.

Thus :

I. The mean area of the $\mathrm{LH}$ peak in ewes from flock II was about $55 \mathrm{p}$. roo of that from ewes in flock I (table 2).

2. The maximum plasma level of $\mathrm{LH}$ in ewes from flock $\mathrm{I}$ was $95.2 \pm 10.5 \mathrm{I} \mathrm{ng} / \mathrm{ml}$ and I02.20 $\pm 6.77 \mathrm{ng} / \mathrm{ml}$, respectively, for one and two ova; in ewes from flock II the values were $55.3 \pm 8.60 \mathrm{ng} / \mathrm{ml}$ and $52.7 \pm 7.65 \mathrm{ng} / \mathrm{ml}$, respectively.

3. The mean durations of LH discharge were respectively $13.9 \pm 0.66 \mathrm{hr}$ and I $1.3 \pm 7.65 \mathrm{hr}$ in flocks I and II.

These results indicate that there is a genetic difference in hypothalamo-hypophyseal function in ewes of the same breed but of different stock. Also we have shown a difference in the duration of the interval between the start of oestrus and the beginning of the $\mathrm{LH}$ surge, depending on whether one or two ova are released. This interval is significantly longer if two ova are discharged $(P<0.02$; table VI).

It is apparent, therefore, that the number of ova released does not depend simply on the magnitude and duration of LH discharge.

\section{RÉFÉRENCES BIBLIOGRAPHIQUES}

Cognie Y., Mariana J. C., Thimonier J., r97o. Étude du moment d'ovulation chez la brebis normale ou traitée par un progestagène associé ou non à une injection de PMSG. Ann. Biol. anim. Bioch. Biophys., 10, I5-24.

FaUl.KNeR L. C., Hopwood M.'L., I967. Clearance of a tritium-labelled progestin in the ewe. J. Anim. Sci., 26, 163-166.

Geschwind I. I., Dewey R., I968. Dynamics of Luteinizing Hormone (LH) secretion in the cycling ewe : a radioimmunoassay study. Proc. Soc. Exp. Biol., 129, 45 I-455.

Goding J. R., Catt K. J., Brown J.M., Kaltenbach $\quad$ C. C., Cumming I. A., Mole B. J., I969. Radioimmunoassay for ovine Luteinizing Hormone. Secretion of Luteinizing Hormone during estrus and followirig estrogen administration in the sheep. Endocrinology, 85, I33-I42.

Jutisz M., Courte C., I968. Hormone lutéinisante (LH) de mouton. I. Obtention à l'état apparemment homogène. Étude physico-chimique et biologique. Gen. Comp. Endocrinol., 11, 562-574.

Mauleon P., Dauzier L., I965. Variations de durée de l'anœstrus de lactation chez les brebis de race Ile de France. Ann. Biol. anim. Bioch. Biophys., 5, I3I-I43.

Mauleon P., Pelletier J., 1964. Variations génétiques du fonctionnement hypophysaire de trois souches de rattes immatures. Relations avec la fertilité. Ann. Biol. anim. Bioch. Biophys., 4, ro5-112. 
Niswender G. D., Roche J. F., Foster D. L., Midgley A. R. Jr., I968. Radioimmunoassay of serum levels of luteinizing hormone during the cycle and early pregnancy in ewes. Proc. Soc. Exp. Biol., 129, 901-904.

Pelletier J., Kann G., Dolais J., Rosselin G., ig68 a. Dosage radioimmunologique de l'hormone lutéinisante plasmatique chez le mouton. Comparaison avec le dosage biologique de LH par la diminution de l'acide ascorbique ovarien, et exemple d'application aux mesures de la LH sanguine chez la brebis. C. R. Acad. Sci. Paris, 266, 2352-2355.

Pelletier J., Kann G., Dolais J., Rosselin G., I968 b. Dosage radioimmunologique de l'hormone lutéinisante plasmatique chez le mouton. Mise au point de la technique de dosage. C. R, Acad. Sci.

Paris, 266, 229I-2294.

Pelletier J., Thimonier J., I969. Étude de la décharge ovulante par dosage radioimmunologique de la LH plasmatique, chez la brebis normale ou traitée par un progestagène. C. R. Acad. Sci. Paris, 268, $573-576$.

RoBinson T. J., r965. Use of progestagene impregnated sponges inserted intravaginally or subcutaneously for the control of the oestrous cycle in the sheep. Nature, 206, 39-4I.

WheAtley I. S., RADFORD H. M., I969. Luteinizing hormone secretion during the oestrous cycle of the ewe determined by radioimmunoassay. J. Reprod. Fert., 18, 2 II-2I4. 